山्لFRANÇAISE

$>\mathrm{DE}$

딜 PÉDAGOGIE
Revue française de pédagogie

Recherches en éducation

179 | avril-juin 2012

Varia

\title{
ROCHEX Jean-Yves \& CRINON Jacques (dir.). La construction des inégalités scolaires. Au cœur des pratiques et des dispositifs d'enseignement
}

Rennes : Presses universitaires de Rennes, 2011, 214 p.

\section{Stanislas Morel}

\section{OpenEdition}

Journals

Édition électronique

URL : http://journals.openedition.org/rfp/3732

DOI : $10.4000 /$ rfp.3732

ISSN : $2105-2913$

Éditeur

ENS Éditions

Édition imprimée

Date de publication : 15 juin 2012

Pagination : 143-144

ISBN : 978-2-84788-379-4

ISSN : 0556-7807

\section{Référence électronique}

Stanislas Morel, «ROCHEX Jean-Yves \& CRINON Jacques (dir.). La construction des inégalités scolaires. Au cœur des pratiques et des dispositifs d'enseignement », Revue française de pédagogie [En ligne], 179 | avril-juin 2012, mis en ligne le 30 novembre 2012, consulté le 22 septembre 2020. URL : http:// journals.openedition.org/rfp/3732 ; DOI : https://doi.org/10.4000/rfp.3732 
sur le cours d'une société qui est loin de représenter un idéal dans son état présent.

L'ouvrage permet en tout état de cause de pointer la faible réflexion sur la définition des contenus d'enseignement en France. La recherche en éducation francophone, en général, a peu de travaux qui mettent en leur centre la question des réformes curriculaires, et ce n'est que depuis la fin du siècle dernier que la sociologie anglaise du curriculum, par exemple, commence à être connue et discutée. À l'heure où se pose sérieusement la question de savoir aussi bien qui doit décider des programmes d'enseignement que celle de savoir sur quels principes les appuyer, le livre de Philippe Perrenoud apporte une contribution stimulante.

Olivier Rey

ENS de Lyon, Institut français de l'Éducation

\section{BIBLIOGRAPHIE}

HABERMAS J. (1973). La technique et la science comme idéologie. Paris : Gallimard.

PERRENOUD P. (1997). Construire des compétences dès l'école. Issy-les-Moulineaux : ESF.

ROCHEX Jean-Yves \& CRINON Jacques (dir.). La construction des inégalités scolaires. Au cœur des pratiques et des dispositifs d'enseignement. Rennes : Presses universitaires de Rennes, 2011, 214 p.

Issu des travaux réalisés dans le cadre du réseau de recherche RESEIDA ${ }^{1}$, cet ouvrage collectif, dirigé par Rochex et Crinon, prend pour objet la construction des inégalités scolaires dans le cadre des situations d'enseignement-apprentissage. Il s'inscrit dans un courant de recherche impulsé en France par les chercheurs du laboratoire CIRCEFT-ESCOL, mais aussi par bien d'autres, dont l'objectif est, pour reprendre le titre d'un colloque qui se tiendra à Lausanne en septembre 2012, la transgression des frontières entre sociologie et didactiques. Si ce genre d'approches suscite actuellement autant d'intérêt, c'est qu'il semble à même de dépasser certains clivages théoriques et méthodologiques qui structurent, cloisonnent et parfois entravent les recherches en sciences de l'éducation, en particulier sur la question des inégalités scolaires. S'appuyant principalement sur des observations longitudinales réalisées entre 2004 et 2006 - la plupart filmées - dans des classes de grande section de maternelle (dont le suivi continue en $\mathrm{CP}$ ) et de $\mathrm{CM} 2$, l'ouvrage est divisé en quatre parties et s'achève sur une conclusion particulièrement dense de Rochex. On présentera ici les principaux mouvements du livre, sans prétendre restituer la complexité et la richesse des onze contributions qu'il rassemble.

Les deux premières parties de cet ouvrage explorent la genèse des inégalités dans les pratiques d'enseignement à travers des processus de différenciation «passive ». L'absence de traitement différencié des élèves dans les situations pédagogiques et, plus spécifiquement, le peu d'attention prêtée à l'inégale distribution des capacités sociocognitives (partie 1) ou socio-langagières (partie 2) nécessaires pour identifier et élaborer les savoirs scolaires visés par les tâches proposées conduisent à des malentendus cognitifs qui favorisent les élèves ayant acquis, le plus souvent en dehors de l'école, les ressources nécessaires à la décontextualisation des connaissances et à leur institutionnalisation en savoirs mémorisables et transposables. Ainsi l'absence de verbalisation des enjeux cognitifs associés à une activité de "mots croisés » en $\mathrm{CP}$ constitue-t-elle un obstacle aux apprentissages attendus, en particulier à ceux, aujourd'hui fondamentaux, qui permettent la maîtrise d'une "littératie étendue » (désignant selon Bautier « les possibilités intellectuelles développées par l'usage heuristique de l'écrit », p. 159). Les élèves en capacité d'effectuer le « saut cognitif » entre la tâche proposée et les savoirs en jeu tirent davantage profit de l'exercice que les élèves les plus faibles de ce point de vue, qui ne perçoivent souvent que le caractère ludique et « ascolaire » de l'activité, et focalisent leur attention sur les tâches matérielles (découpage ou collage).

La troisième partie met en lumière un deuxième type de processus de différenciation, cette fois « active », lié à la prise en compte par les enseignants des différences, réelles ou supposées, de leurs élèves. L'adaptation des tâches, des supports et des modes de travail (à travers, par exemple, la mise en place d' " ateliers » par niveau au sein de la classe), l'établissement de contrats didactiques différenciés modulant les exigences et, enfin, le recours à des «modes de cadrage » de l'activité plus ou moins souples en fonction des élèves, tendent, à l'insu des enseignants, à renforcer les inégalités en conduisant les élèves d'une même classe à fréquenter « des univers de travail et de savoir différenciés, et inégalement productifs en termes d'activité intellectuelle et d'apprentissage potentiels » (p. 178). De ce fait, les évaluations satisfaisantes au cours des premières années de la scolarité peuvent s'avérer trompeuses car elles ne correspondent pas nécessairement à de réels apprentissages, les décalages se donnant à voir ultérieurement, notamment lors des changements de cycles. Par ailleurs, surtout dans les contextes marqués par la présence importante d'enfants issus des classes populaires précarisées, l'attention des enseignants se focalise non pas tant sur les différences d'apprentissage que sur celles relatives au comportement (attitude en classe, 
rapport au travail), ce qui contribue à reléguer au second plan la question centrale des enjeux et des contenus de savoirs.

La récurrence du phénomène d' « invisibilisation » des savoirs scolaires (mentionné dans la quasi-totalité des contributions) laisse penser qu'il ne relève pas seulement de pratiques indépendantes, mais qu'il est l'expression d'un genre pédagogique actuellement dominant à l'école, que la quatrième partie de l'ouvrage s'attache à caractériser. Se situant à un niveau de conceptualisation intermédiaire, les contributions de cette partie appréhendent les processus de différenciation observés en classe comme l'actualisation, dans des situations pédagogiques, de transformations sociales et scolaires plus générales. L'analyse de l'évolution des « dispositifs pédagogiques », de la « forme scolaire », des « discours pédagogiques » ou des « curriculums » permet ainsi de resituer les processus étudiés dans un contexte socio-historique plus global, relativement indépendant des interactions pédagogiques observées pendant l'enquête. Cette montée en généralité permet aux auteurs de faire apparaître certaines logiques vectrices d'inégalités, actuellement à l'œuvre à l'école primaire. Il s'agit d'abord d'une focalisation sur les tâches au détriment des savoirs dont l'acquisition est pourtant à la base de l'évaluation et de la sélection scolaires. Sont ensuite soulignés les effets de deux types de brouillages cognitifs, aussi bien pour les élèves que pour les enseignants : le premier induit par l'assouplissement de la séparation entre les différents domaines de savoirs scolaires (décloisonnement des disciplines et attentes implicites en termes de « compétences » transversales), le second par la mobilisation de plus en plus fréquente dans le cadre scolaire d'expériences et de discours non scolaires en lieu et place de situations et de modes de verbalisation explicitement en lien avec les savoirs scolaires visés. Les inégalités résultent enfin des manières dont les enseignants contrôlent la communication pédagogique, associant tantôt un cadrage fort et une focalisation des élèves faibles sur des tâches fragmentées et peu exigeantes intellectuellement, tantôt un cadrage faible et une pédagogie implicite laissant en partie aux élèves le travail d'élaboration des savoirs en jeu à partir de la mise en activité proposée. Ces trois logiques sont productrices d'inégalités dans la mesure où elles défavorisent les élèves les moins familiers avec les savoirs scolaires.

Proposant, comme on a essayé de le montrer, une approche des inégalités scolaires qui multiplie les angles d'entrée dans la question et les niveaux d'analyse, cet ouvrage très riche peut toujours être critiqué pour son caractère incomplet. On s'efforcera de ne pas céder à cette facilité et, pour conclure cette recension, on formulera deux remarques sur le cœur du projet. La première concerne le croisement des approches didactiques et sociologiques, dont le tissage est loin d'être évident du côté des contributions des didacticiens. Ces derniers, en effet, semblent focalisés sur les interactions pédagogiques en classe et différencient peu les élèves (si ce n'est les «bons » et les «faibles »). De leur côté, les sociologues semblent s'être davantage approprié les outils des didacticiens, ce qui leur permet d'éclairer l'étude des interactions pédagogiques par une perspective sociologique (mise en relation de ces interactions avec les propriétés sociales des acteurs impliqués ou, grâce à des niveaux de conceptualisation intermédiaires, avec des transformations sociales plus générales). Cette asymétrie, qui donne le beau rôle aux sociologues, peut-elle être généralisée ou, à l'inverse, n'est-elle qu'une image isolée ne rendant pas compte des formes d'appropriation de la sociologie par les didacticiens ?

La deuxième porte sur l'articulation entre la présentation des observations en classe, les analyses se situant à d'autres niveaux de conceptualisation et, enfin, intention peu explicitée dans le livre, le rappel des principaux résultats des recherches menées au sein du laboratoire CIRCEFT-ESCOL. Si ce triple objectif donne toute sa profondeur au projet, on peut cependant regretter que, dans certaines contributions - en particulier de la quatrième partie -, la place centrale accordée à l'exposition des transformations de l'institution scolaire ou au rappel des résultats consolidés au fil du temps réduise parfois à la portion congrue la présentation des résultats de cette nouvelle recherche, cantonnés au rang de vignettes illustratives alors qu'ils sont pourtant censés être au cœur de l'analyse et de l'administration de la preuve.

En définitive, l'ouvrage est cependant convaincant. L'approche à la fois « relationnelle et contextuelle » (p. 9) est à l'origine d'une série de dépassements. Montrant la complémentarité d'approches théoriques parfois opposées (par exemple celles de Bourdieu et de Bernstein), croisant les regards disciplinaires, situant l'analyse à la fois sur le plan des interactions pédagogiques et à des niveaux de conceptualisation intermédiaires ou, enfin, recourant à des approches diachroniques et synchroniques, ce travail, au-delà des résultats exposés ci-dessus, ouvre également de nombreuses perspectives de recherche, en particulier pour tous ceux qui s'intéressent au rapprochement entre didactique et sociologie.

Stanislas Morel Université Jean-Monnet-Saint-Étienne, Éducation, cultures, politiques

\section{NOTE}

1 Réseau de recherche sur la socialisation, l'enseignement, les inégalités et les différenciations dans les apprentissages créé en 2001. 